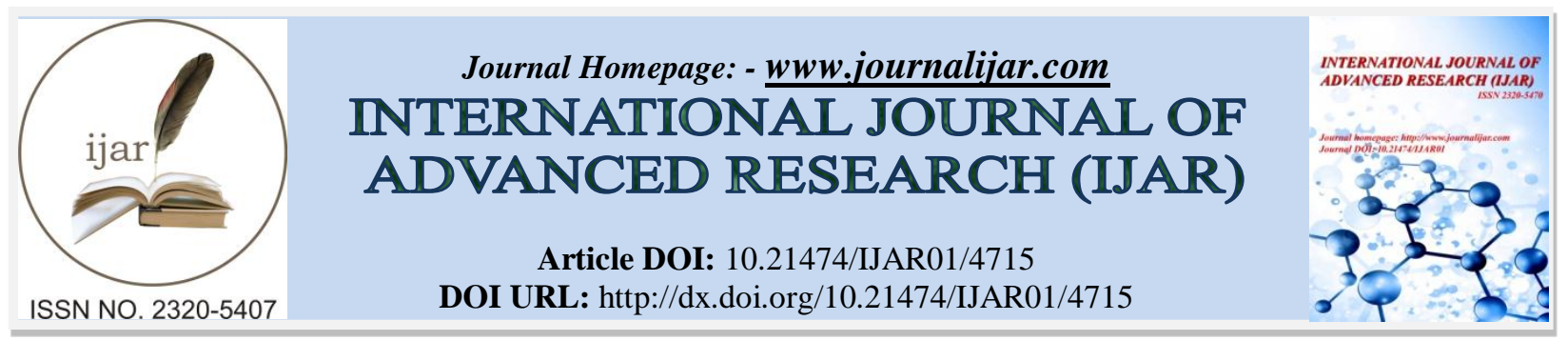

RESEARCH ARTICLE

\title{
USING OF HYDRAULIC JUMP CHARACTERISTICS AS CRITERION FOR ENERGY DISSIPATION IN RECTANGULAR SHAPED GRAVEL GABION WEIR.
}

Fadhil Mohammed Dhahir ${ }^{1}$ and Ali Mecki Mohammed Hasan ${ }^{2}$.

1. Kerbala Technical Institute, AL-Furat Al-Awsat Technical University, 56001 Kerbala, Iraq.

2. Operating Department, Directorate of Water Resources in Kerbala, Kerbala, Iraq.

\section{Manuscript Info}

Manuscript History

Received: 3 May 2017

Final Accepted: 5 June 2017

Published: July 2017

Key words:-

weirs; hydraulic characteristics; energy dissipation; gabion weir; hydraulic jump; energy loss.

\section{Abstract}

Dissipation of energy of the flow by using of weirs can be accomplish in many ways, depending basically on the type of weir. The concept of through and overflow for porous weirs can be use purposively to explain many phenomena such as, dissipating the energy of incoming flow which the flowing of water through the body of the weir contributes in considerable percentage of it, and using of another concepts to represent the dissipated energy in the downstream side of the weir as criterions for energy dissipation. This paper aims to study the dissipated amount of energy for gravel gabion weir by choosing of the difference in energy between upstream and downstream sides of the weir, and the distance of formation of the hydraulic jump as criterions to represent the energy dissipation. The general conclusion shows that both criterions are directly proportional with each other for all series of test runs, while every parameter, fixed of measured, such as length of the weir and diameter of gravel sample has an undular effect on both criterions.

Copy Right, IJAR, 2017. All rights reserved.

\section{Introduction:-}

A lot of studies and researches have been carried out to study and discuss various topics concerning flow behavior in weirs. In order to coherent these topics, it's important to study and realize hydraulic structures from different aspects such as, study the hydraulic characteristics of flow over solid weirs, flow through and over pervious weirs, energy loss and energy dissipation of flow in weirs. This paper aims to study the dissipated energy of flow for the rectangular shape of "Gravel Gabion Type" weir.

\section{Hydraulic Characteristics of Flow:-}

Studying of the characteristics of flow through and over weirs consider as the main and one of priority objects that can be discuss to explain different types of flow phenomena that can occur in various water resources projects. Studying of hydraulic characteristics of flow helps firstly, to find a proper analogy to create a comparison between solid and rockfill weirs in over flow case. Secondly, make a possibility of use empirical equations to understand the behavior of flow through and over rockfill structures [6], [15], and [16]. Examples of hydraulic behavior in weirs are much more than correspond herein, and differ according to the aim of study. Such examples as describing various relationships for non-Darcy flow in rockfill material, by considering the friction factor- Reynolds number, and hydraulic gradient-bulk seepage velocity theoretical relationships as main relations. In addition, making a comparison with various formulas for rockfill available from literature by computer simulation [3]. Reanalyzing the 
characteristics of the free surface undulations downstream the first wave crest for undular flows and undular hydraulic jumps, by depending the relationship between wave amplitude and the approach flow Froude number [5]. Investigation of flow over gabion weirs by Performing a series of laboratory experiments, and using the relationship between the parameters such as, discharge, upstream and downstream water depths, weir height, weir length, and gabion filling material [7]. Determination of flow conditions through and over rockfill embankment, by taking the free surface flow regime as spatially varied, and the seepage flow regimes as non-Darcian for two conditions of flow. The partial overtopping and complete overtopping [8]. Finally, studying the hydrodynamics of a rubble mound weir in both theoretical and experimental sides. Performing a one-dimensional analysis on a steady non-uniform flow through the weir. Where the discharge as a function of related parameters, such as flow depths on upstream and downstream faces of the weir, porosity, weir length, and grain diameter of the rubble mound [12].

\section{Energy Loss of Flow in Porous Weirs:-}

A complete understanding of the energy losses in porous weirs is necessary to find the proper relationship for this topic. Reference [4] studied the energy loss at drops by investigating the dissipated energy of three drops with different heights as physical models made of Plexiglas, and installed in two laboratory flumes. By using an artificial neural networks approach. He developed an explicit procedure to calculate the resulted energy loss by NeuroSolutions. His results showed that the energy dissipation in drops depend on the drop height and discharge. The predicted relative energy dissipation varied from $10 \%$ to $93.4 \%$. Finally, the energy loss at drops is mainly due to mixing of the jet with the pool behind the jet that causes air bubbles entrainment in the flow. Reference [9] studied the energy loss through porous dykes with different shapes and materials experimentally. He used the friction factor - Reynolds number relationship as a representation for the head loss through the body of the dyke. In addition, the influence of another parameters such as stone shape and porosity on the relationship. He noticed the contrary attitude between parameters in his relationship, and concluded that the friction factor for the same Reynolds numbers for the used shapes were in agreement with one another, and affected by stone angularity and porosity. Reference [18] evaluated an empirical equations to estimate the hydraulic parameters of the non-linear flow through coarse porous media. He used a series of independent data collected in the laboratory, resulted from testing three different relatively uniform soils with three random samples drawn from each material. The equation was performed for the physical characteristics such as size distribution, porosity, and shape factor for all samples. The statistical analysis showed that the performed equation can give good results compared with literature formulas that used in the same field measurements representation, and show acceptable performance. Reference [20] proposed a head loss equation for flow through rockfill to be acceptable for a wide range of rock particle size. In addition, using it for prediction of flow transmissibility of rockfill structures by selecting the Forschheimer equation to describe the head loss property of flow through rockfill. The study was summarized by considering Forschheimer equation as the best formula that can be applied to rockfill particle size from $5 \mathrm{~mm}$ to about $100 \mathrm{~mm}$, and to predict flow transmissibility of rockfill structures.

\section{Experimental Work:-}

All tests were carried out in the hydraulic laboratory of College of Engineering of the Babylon University in Iraq. The laboratory has a tilting flume of $10 \mathrm{~m}$ length, with $0.3 \mathrm{~m}$ width and $0.5 \mathrm{~m}$ height. The bed of flume was fabricated from iron plates and the flume side walls were made of anti-crush glass supported by stainless steel bars. The physical models that used have lengths of 40,60, 80, 100, and $120 \mathrm{~cm}$, and named as GGW.No.1, (Gravel Gabion Weir Number 1), GGW.No.2, GGW.No.3, GGW.No.4, and GGW.No5 respectively. All models have constant cross-section, (width of $30 \mathrm{~cm}$ and height of $40 \mathrm{~cm}$ ). While the gravel samples that used as filling material for the weir models were five monosized gravel samples with diameters (9.5-14), (14-19), (19-25), (25-37.5), and (37.5-50) mm, and numbered as G.S.No.1, (gravel sample number1), G.S.No.2, G.S.No.3, G.S.No.4, and G.S.No.5 respectively. The frame of GGW was made of thin steel plated bars, covered by a wire mesh, and fixed inside the flume by silicone glue. A photo of GGW.No.1 with G.S.No.1 illustrated in Fig. 1. A centrifugal pump having a rated capacity of $40 \mathrm{l} / \mathrm{s}$ was used to deliver flow to the flume. Two movable carriages with point gages were mounted on brass rail at the top of flume sides which have accuracy of $0.1 \mathrm{~mm}$ to measure the depths of water. The first was located at the upstream side of GGW and measures the upstream water depth at equal distances $0.0,0.2,0.4,0.6$, 0.8 , and $1.0 \mathrm{~m}$ before GGW during the single test run. The other was at the downstream side of GGW and measures the downstream water depths before and after the hydraulic jump location during the single test run. A total of 194 test runs were carried out and varied between minimum and maximum values of discharges recorded from 0.70 to $14.0251 / \mathrm{s}$ respectively. 
The Concept of Energy for GGW:-

The magnitude of energy at the upstream side of GGW can be calculated by:

$$
E_{u}=Z_{u}+y_{u}+\frac{v_{u}^{2}}{2 g}=Z_{u}+y_{u}+\frac{q^{2}}{2 g y_{u}^{2}}
$$

where $E_{u}$ is the magnitude of energy at the upstream side of GGW (m), $Z_{u}$ is the elevation head directly under $y_{u}(\mathrm{~m})$, $y_{u}$ is the upstream water depth of GGW (m), $v_{u}$ is the velocity of flow at the upstream side of GGW (m/s), $g$ is the gravitational acceleration $\left(\mathrm{m} / \mathrm{s}^{2}\right)$, and $q$ is the discharge per unit width $\left(\mathrm{m}^{3} / \mathrm{s} / \mathrm{m}\right)$. While, the magnitude of energy at the downstream side of GGW can be calculated by:

$$
E_{l}=y_{1}+\frac{v_{1}^{2}}{2 g}=y_{1}+\frac{q^{2}}{2 g y_{1}^{2}}
$$

where $E_{l}$ is the magnitude of energy at the downstream side of GGW (m), $y_{l}$ is the depth of water at downstream side of GGW before the hydraulic jump, and $v_{l}$ is the velocity of flow at the downstream side of GGW (m/s). The difference in energy between the upstream and downstream sides of GGW, $\triangle E$, may be calculated by:

$$
\Delta E=E_{u}-E_{1}
$$

\section{Results of Representation of Energy Dissipation in GGW:-}

Previous researchers have used different relations to represent the dissipated energy in hydraulic structures by developing empirical equations for hydraulic jump as criterion of energy dissipation [1], [10], [11], [19], [22], and [23]. The present paper aims to view the direct relationship between $\Delta \mathrm{E}$, and the distance of hydraulic jump, DHJ, formed away. From downstream toe of GGW, as criterions for representation of energy dissipation.

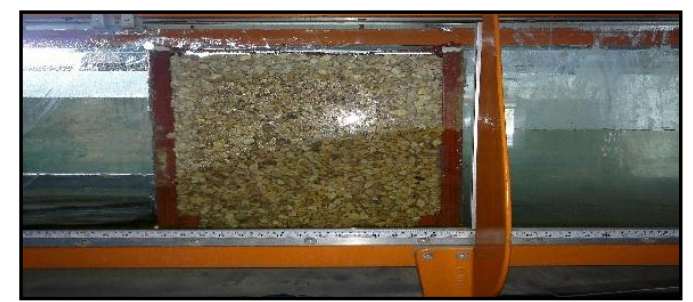

Fig. 1:- A photo of GGW.No.1 with G.S.No.1.

Figures 2, 3, 4, 5, and 6 show the relationship above for five different lengths of GGW used in this paper. These figures show that the best form of equation of trend line which represents the relationship $\Delta \mathrm{E}-\mathrm{DHJ}$ was the exponential form for Figs. 2, 3, 4, and 5, and the multi linear for Fig. 6 by using of "Microsoft - Excel" computer program as in (4) and (5) below :

\begin{tabular}{|c|c|}
$D H J=c_{1} e^{c 2 \Delta E}$ & (4) \\
$D H J=h_{1} \Delta E^{2}+h_{2} \Delta E+h_{3}$ & (5) \\
\hline
\end{tabular}

where $c_{1}, c_{2}, h_{1}, h_{2}$, and $h_{3}$ are coefficients. Table I presents the values of coefficients $c_{1}, c_{2}, h_{1}, h_{2}$, and $h_{3}$. Generally, for all figures, $\Delta \mathrm{E}$ values are directly proportional with $\mathrm{DHJ}$ values. This behavior comes from transforming of the potential energy at the upstream side of GGW to kinetic energy at its downstream side, which causes to increase the distance of formation of hydraulic jump from the toe of GGW. Fig. 2 shows that increasing of both values of $\Delta \mathrm{E}$ and diameter of gravel sample used $\mathbf{d}$ has no arresting effect on the DHJ value at the minimum and medium value of $\Delta \mathrm{E}$, and that appears clearly by noticing the intersection and approach of trend lines from each other for G.S.No.2, 3, 4, and 5 till it gets $\Delta \mathrm{E}$ equal to $0.175 \mathrm{~m}$, where the diverge in trend lines starts noticeably after $\Delta \mathrm{E}$ equal to $0.210 \mathrm{~m}$, which refer to start the increasing in values of DHJ directly with increasing both values of $\Delta \mathrm{E}$ and $\mathbf{d}$. Fig. 3 explain that the trend lines approach for each other for minimum values of $\Delta \mathrm{E}$, which refer to approach of DHJ values for all gravel samples at these values, but this approach disappears by the diverge of trend lines with increasing both values of $\Delta \mathrm{E}$ and DHJ. Increasing of $\mathbf{d}$ value shows undular effect on both criterions. Fig. 4 shows that the approach of $\mathrm{DHJ}$ values from each other for different gravel samples at the minimum values of $\Delta \mathrm{E}$ by noticing the intersection and approach of trend lines from each other for these values. The gradation of gravel samples appears clearly on both G.S.No.1 and G.S.No.2, where values of DHJ decrease by increasing both values of $\mathbf{d}$ and $\Delta \mathrm{E}$, but the gradation has no clear effect on the last three gravel samples, G.S.No.3, 4, and 5, and the trend lines show the limited effect of increasing in $\Delta \mathrm{E}$ values on values of DHJ. Fig. 5, as in figures above, illustrates that the approach of trend lines from each other is clear which refers to approach of DHJ values from each other for minimum values of $\Delta \mathrm{E}$, and the clearance of intersection of trend lines at these values. The values of DHJ increases by increasing of 
$\Delta \mathrm{E}$ values and decreasing of $\mathbf{d}$ values. Fig. 6 shows that the behavior of trend lines at minimum values of $\Delta \mathrm{E}$ is as in figures above for the last three gravel samples, G.S.No.3, 4, and 5. The values of DHJ for G.S.No.1 and G.S.No.2 increase by increasing of $\Delta \mathrm{E}$ values noticeably. The effect of increasing of $\Delta \mathrm{E}$ values on DHJ values is light for minimum and medium values of $\Delta \mathrm{E}$, but it becomes good and clear for high values of $\Delta \mathrm{E}$. All these variations happen with decreasing of $\mathbf{d}$ value. To identify the effect of both of applied discharge and $\mathbf{d}$ precisely on both values of $\Delta \mathrm{E}$ and $\mathrm{DHJ}$, three values of applied discharge were chosen for every gravel sample used, and their values were the minimum value of discharge, $\left(\mathrm{q}_{\min }=0.7 \mathrm{l} / \mathrm{s}\right)$, the comparison value of discharge, $\left(\mathrm{q}_{\mathrm{cmpr}}=3.6 \mathrm{l} / \mathrm{s}\right)$, and the maximum value of discharge measured within the series of test runs that carried out on each gravel sample, $\left(\mathrm{q}_{\max }\right)$. Table II presents the values of both of $\Delta \mathrm{E}$ and DHJ for the chosen values of applied discharge, gravel samples, and lengths of GGW. For GGW.No.1, the hydraulic jump approaches toward the toe of GGW for the three values of applied discharge, $\left(\mathrm{q}_{\min }, \mathrm{q}_{\mathrm{cmpr}}\right.$, and $\left.\mathrm{q}_{\max }\right)$, as the value of $\mathbf{d}$ starts to increase, while the value of $\Delta \mathrm{E}$ decreases. For GGW.No.2, the hydraulic jump approaches, (for all values of $\mathbf{d}$ ), from the toe of GGW, (but with a little difference between values of hydraulic jump), at the $\mathrm{q}_{\mathrm{min}}$, and this approach appears clearly at $\mathrm{q}_{\mathrm{cmpr}}$, the approach starts to fluctuate within a range of $(1.35-1.565) \mathrm{m}$ for $\mathrm{q}_{\max }$ with increasing $\mathbf{d}$ values. For GGW.No.3, the gradation of $\mathbf{d}$ has no effect on the value of DHJ at $\mathrm{q}_{\mathrm{min}}$, while at $\mathrm{q}_{\mathrm{cmpr}}$ the hydraulic jump starts to approach toward the toe of GGW with increasing of $\mathbf{d}$ with noticing that the values of DHJ for the last three values of $\mathbf{d}$ lies in a short range, (in other word the effect of gradation starts to vanish at this range). For $\mathrm{q}_{\max }$, the value of DHJ fluctuates with increasing of value of $\mathbf{d}$. All the changes which were happened in GGW.No.3 continue to include GGW.No.4. for both criterions. For GGW.No5 and $\mathrm{q}_{\min }$, there is no existence of the hydraulic jump for all $\mathbf{d}$ values, but the hydraulic jump starts to appear at $\mathrm{q}_{\mathrm{cmpr}}$ where it approaches to the toe of GGW as the value of $\mathbf{d}$ decreases, and this effect appears regularly at $\mathrm{q}_{\max }$. The value of $\Delta \mathrm{E}$ decreases at both $\mathrm{q}_{\min }$ and $\mathrm{q}_{\mathrm{cmpr}}$, but fluctuates at $\mathrm{q}_{\max }$ with increasing of value of $\mathbf{d}$ for GGW.No.2, 3, and 4 respectively. While the $\Delta \mathrm{E}$ measured at $\mathrm{q}_{\min }$ for GGW.No.5 shows a fluctuation in their values, but this fluctuation disappears at both $\mathrm{q}_{\mathrm{cmpr}}$ and $\mathrm{q}_{\max }$, where the value of $\Delta \mathrm{E}$ starts to decrease by increasing of the value of $\mathbf{d}$.

\section{Dimensional Analysis:-}

In general, energy dissipation of the flow passing through and over GGW depends on hydraulic and geometric variables. For rectangular shape of GGW, these variables can be functionally expressed as:

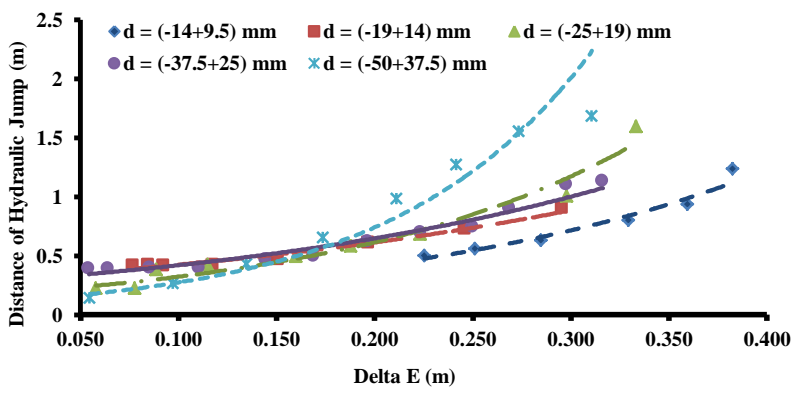

Fig. 2:- DHJ- $\Delta$ E relationship for GGW.No.1

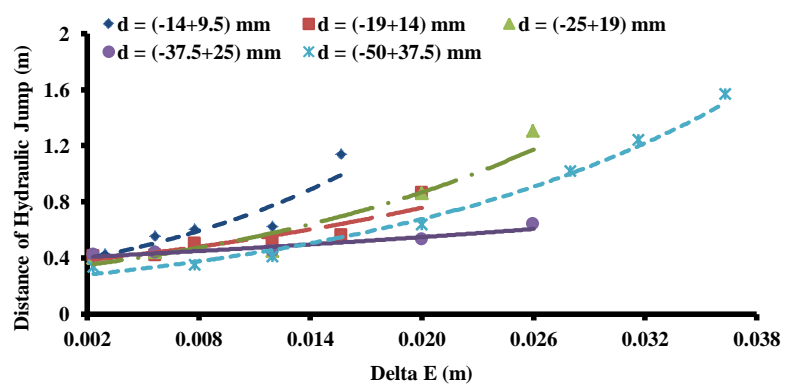

Fig. 3:- DHJ- $\Delta$ E relationship for GGW.No.2. 


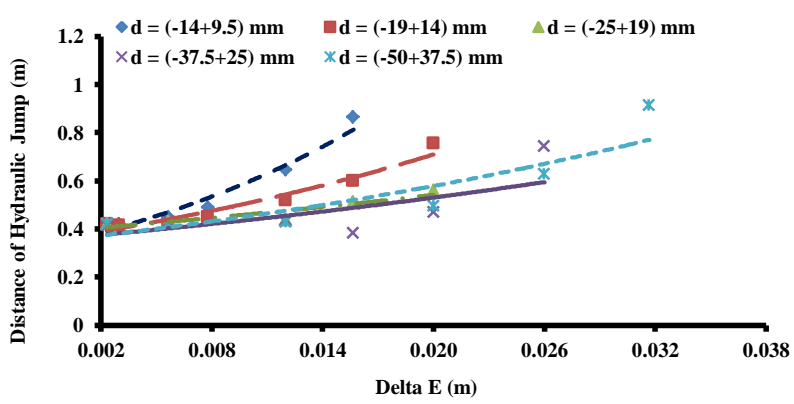

Fig. 4:- DHJ- $\Delta$ E relationship for GGW.No.3.

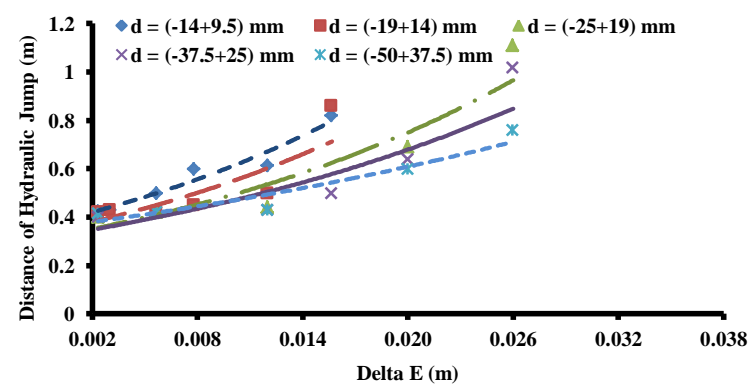

Fig. 5:- DHJ- $\Delta$ E relationship for GGW.No.4.

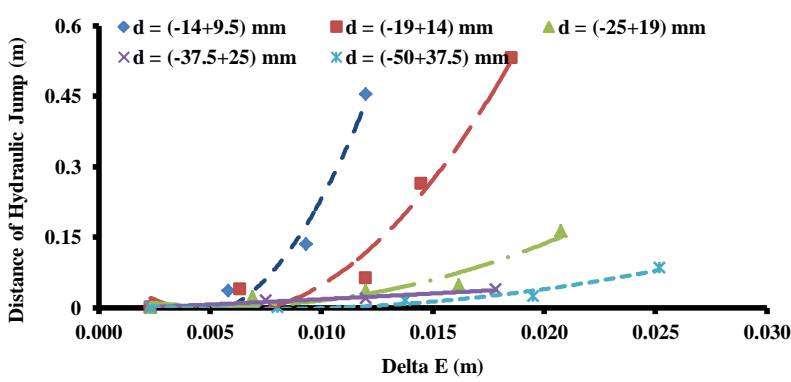

Fig. 6:- DHJ- $\Delta$ E relationship

Table I:- Values of coefficients $c_{1}, c_{2}, h_{1}, h_{2}$, and $h_{3}$ of DHJ- $\Delta$ E relationship.

\begin{tabular}{|c|c|c|c|c|c|}
\hline LT (cm) & $\mathbf{d}(\mathbf{m m})$ & $\mathbf{c}_{\mathbf{1}}$ & $\mathbf{c}_{\mathbf{2}}$ & - & $\mathbf{R}^{\mathbf{2}}$ \\
\hline \multirow{4}{*}{40} & $9.5-14$ & 0.14 & 5.434 & - & 0.965 \\
\cline { 2 - 6 } & $14-19$ & 0.293 & 3.681 & - & 0.968 \\
\cline { 2 - 6 } & $19-25$ & 0.168 & 6.457 & - & 0.945 \\
\cline { 2 - 6 } & $25-37.5$ & 0.271 & 4.349 & - & 0.957 \\
\cline { 2 - 6 } & $37.5-50$ & 0.100 & 9.968 & - & 0.964 \\
\hline \multirow{6}{*}{60} & $9.5-14$ & 0.342 & 69.36 & - & 0.943 \\
\cline { 2 - 6 } & $14-19$ & 0.355 & 37.94 & - & 0.965 \\
\cline { 2 - 6 } & $19-25$ & 0.316 & 50.42 & - & 0.912 \\
\cline { 2 - 6 } & $25-37.5$ & 0.394 & 16.59 & - & 0.979 \\
\hline \multirow{3}{*}{80} & $37.5-50$ & 0.255 & 48.83 & - & 0.960 \\
\cline { 2 - 6 } & $9.5-14$ & 0.345 & 54.60 & -948 \\
\cline { 2 - 6 } & $14-19$ & 0.364 & 33.33 & - & 0.866 \\
\cline { 2 - 6 } & $19-25$ & 0.391 & 16.12 & - & 0.815 \\
\cline { 2 - 6 } & $25-37.5$ & 0.360 & 19.19 & - & 0.948 \\
\hline
\end{tabular}




\begin{tabular}{|c|c|c|c|c|c|}
\hline & 14-19 & 0.346 & 46.08 & - & 0.749 \\
\hline & $19-25$ & 0.322 & 42.16 & - & 0.897 \\
\hline & $25-37.5$ & 0.322 & 37.18 & - & 0.840 \\
\hline & $37.5-50$ & 0.360 & 26.19 & - & 0.898 \\
\hline \multirow[t]{6}{*}{120} & & $\mathbf{h}_{1}$ & $\mathbf{h}_{2}$ & $\mathbf{h}_{3}$ & $\mathbf{R}^{2}$ \\
\hline & $9.5-14$ & 7663 & -65.82 & 0.123 & 0.973 \\
\hline & 14-19 & 3241 & -36.43 & 0.088 & 0.966 \\
\hline & $19-25$ & 649.9 & -7.410 & 0.024 & 0.913 \\
\hline & $25-37.5$ & 15.81 & 2.028 & -0.003 & 0.977 \\
\hline & $37.5-50$ & 253.6 & -3.592 & 0.009 & 0.953 \\
\hline
\end{tabular}

where $y_{2}$ is the depth of water at the downstream side of GGW after the hydraulic jump (m), LT is the length of GGW $(\mathrm{m}), \mathrm{S}_{\mathrm{f}}$ is the shape factor, and $\rho$ the mass density $\left(\mathrm{kg} / \mathrm{m}^{3}\right)$. The shape factor can be computed from:

$$
S_{f}=\frac{n_{3}}{\sqrt{n_{1} * n_{2}}}
$$

where $\mathrm{n}_{1}, \mathrm{n}_{2}$, and $\mathrm{n}_{3}$ are the longest, medium, and shortest dimensions of gravel particles respectively. From (1), (2), and (3), its notice that $E_{u}$ depends on $Z_{u}$ and $y_{u}, Z_{u}$ depends on the slope of the laboratory flume which is fixed, and $E_{l}$ depends on $y_{1}$. So, (6) can be re-written as

$$
f\left\{q, \Delta E, L T, \boldsymbol{d}, D H J, S_{f}, \rho, g\right\}=0
$$

Using of Buckingham $\mathrm{Pi}$ - Theorem consider as an effective way to represent the relationships of parameters functionally in dimensionless mode, [2], [13], and [17]. The representation of energy dissipation by the hydraulic jump can be expressed as non-dimensional parameters as follows:

$$
\frac{D H J}{\boldsymbol{d}}=f\left\{\frac{\Delta E}{\boldsymbol{d}}, \frac{q}{\sqrt{g} * \boldsymbol{d}^{1.5}}, \frac{L T}{\boldsymbol{d}}, S_{f}\right\}
$$

\begin{tabular}{|c|c|c|c|c|}
\hline \multirow{2}{*}{ Criterion } & \multirow{2}{*}{ d (mm) } & \multicolumn{3}{|c|}{ Discharge (1/s) } \\
\hline & & 0.7 & 3.6 & $q_{\max }$ \\
\hline \multirow[t]{5}{*}{ DHJ (m) for GGW.No.1 } & $9.5-14$ & 0.500 & 1.585 & 3.000 \\
\hline & 14-19 & 0.417 & 0.610 & 2.200 \\
\hline & $19-25$ & 0.220 & 0.490 & 1.765 \\
\hline & $25-37.5$ & 0.390 & 0.470 & 1.720 \\
\hline & $37.5-50$ & 0.140 & 0.430 & 1.710 \\
\hline \multirow{5}{*}{$\begin{array}{c}\Delta E(m) \\
\text { for GGW.No.1 }\end{array}$} & $9.5-14$ & 0.225 & 0.397 & 0.406 \\
\hline & $14-19$ & 0.077 & 0.197 & 0.374 \\
\hline & $19-25$ & 0.058 & 0.160 & 0.357 \\
\hline & $25-37.5$ & 0.054 & 0.144 & 0.345 \\
\hline & $37.5-50$ & 0.055 & 0.135 & 0.338 \\
\hline \multirow[t]{5}{*}{ DHJ (m) for GGW.No.2 } & $9.5-14$ & 0.400 & 0.620 & 1.405 \\
\hline & 14-19 & 0.415 & 0.530 & 1.445 \\
\hline & $19-25$ & 0.400 & 0.450 & 1.350 \\
\hline & $25-37.5$ & 0.425 & 0.450 & 1.425 \\
\hline & $37.5-50$ & 0.335 & 0.410 & 1.565 \\
\hline \multirow{5}{*}{$\begin{array}{c}\Delta E(m) \\
\text { for } G G W . N o .2\end{array}$} & $9.5-14$ & 0.107 & 0.285 & 0.371 \\
\hline & 14-19 & 0.088 & 0.235 & 0.378 \\
\hline & $19-25$ & 0.069 & 0.184 & 0.386 \\
\hline & $25-37.5$ & 0.064 & 0.165 & 0.375 \\
\hline & $37.5-50$ & 0.051 & 0.134 & 0.334 \\
\hline \multirow[t]{3}{*}{ DHJ (m) for GGW.No.3 } & $9.5-14$ & 0.415 & 0.645 & 1.150 \\
\hline & 14-19 & 0.420 & 0.520 & 0.755 \\
\hline & $19-25$ & 0.420 & 0.440 & 0.755 \\
\hline
\end{tabular}

Table II:- Values of $\Delta \mathrm{E}$, DHJ, q, and $\mathbf{d}$ for GGW.No.1, 2, 3, 4, and 5. 


\begin{tabular}{|c|c|c|c|c|}
\hline & $25-37.5$ & 0.415 & 0.440 & 1.200 \\
\hline & $37.5-50$ & 0.420 & 0.430 & 1.360 \\
\hline \multirow{5}{*}{$\begin{array}{c}\Delta E(m) \\
\text { for } G G W . N o .3\end{array}$} & $9.5-14$ & 0.095 & 0.267 & 0.377 \\
\hline & $14-19$ & 0.071 & 0.236 & 0.350 \\
\hline & $19-25$ & 0.074 & 0.215 & 0.354 \\
\hline & $25-37.5$ & 0.069 & 0.179 & 0.364 \\
\hline & $37.5-50$ & 0.064 & 0.163 & 0.360 \\
\hline \multirow[t]{5}{*}{ DHJ (m) for GGW.No.4 } & $9.5-14$ & 0.425 & 0.615 & 1.085 \\
\hline & $14-19$ & 0.420 & 0.500 & 1.400 \\
\hline & $19-25$ & 0.400 & 0.440 & 1.720 \\
\hline & $25-37.5$ & 0.400 & 0.430 & 1.61 \\
\hline & $37.5-50$ & 0.415 & 0.430 & 1.330 \\
\hline \multirow{5}{*}{$\begin{array}{c}\Delta E(\mathrm{~m}) \\
\text { for GGW.No.4 }\end{array}$} & $9.5-14$ & 0.119 & 0.331 & 0.374 \\
\hline & $14-19$ & 0.095 & 0.275 & 0.394 \\
\hline & $19-25$ & 0.077 & 0.230 & 0.392 \\
\hline & $25-37.5$ & 0.068 & 0.195 & 0.383 \\
\hline & $37.5-50$ & 0.066 & 0.169 & 0.357 \\
\hline \multirow[t]{5}{*}{ DHJ $(\mathrm{m})$ for GGW.No.5 } & $9.5-14$ & zero & 0.455 & 1.280 \\
\hline & 14-19 & zero & 0.062 & 1.166 \\
\hline & $19-25$ & zero & 0.035 & 0.740 \\
\hline & $25-37.5$ & zero & 0.020 & 0.635 \\
\hline & $37.5-50$ & zero & zero & 0.265 \\
\hline \multirow{5}{*}{$\begin{array}{c}\Delta E(m) \\
\text { for GGW.No.5 }\end{array}$} & $9.5-14$ & 0.094 & 0.311 & 0.397 \\
\hline & $14-19$ & 0.103 & 0.291 & 0.374 \\
\hline & $19-25$ & 0.049 & 0.261 & 0.389 \\
\hline & $25-37.5$ & 0.054 & 0.223 & 0.365 \\
\hline & $37.5-50$ & 0.024 & 0.181 & 0.346 \\
\hline
\end{tabular}

In order to represent the relationship between the dimensionless parameters above for every single value of length of GGW, the dimensionless parameter $\frac{L T}{\boldsymbol{d}}$ should be eliminated. The term $\left(\frac{q}{\sqrt{g} * \boldsymbol{d}^{1.5}}\right)$ can be written as $\left(\frac{F r_{g} \cdot y_{u}}{\boldsymbol{d}}\right)$ where $\mathrm{Fr}_{\mathrm{g}}$ represents Froude Number of gravel used. Equation (8) will become

$$
\frac{D H J}{\boldsymbol{d}}=f\left\{\frac{\Delta E}{\boldsymbol{d}}, F r_{g}, \frac{y_{u}}{\boldsymbol{d}}, S_{f}\right\}
$$

By using of multi-linear regression for the data set to correlate the dependent parameters with other independent ones, [14] and [21], the resulted formula can expressed by:

$$
D H J=K_{1} \cdot \Delta E^{K_{2}} \cdot \mathrm{Fr}_{g}{ }^{K_{3}} \cdot y_{u}{ }^{K_{4}} \cdot \boldsymbol{d}^{K_{5}} \cdot S_{f}{ }^{K_{6}}
$$

where $\mathrm{K}_{1}, \mathrm{~K}_{2}, \mathrm{~K}_{3}, \mathrm{~K}_{4}, \mathrm{~K}_{5}$, and $\mathrm{K}_{6}$ are coefficients depending on $\mathbf{d}$. Table III presents the values of $\mathrm{K}$ coefficients that used in (11).

Table III:- Values of $\mathrm{K}$ coefficients.

\begin{tabular}{|c|c|c|c|c|c|}
\hline \multirow{2}{*}{ K constants } & \multicolumn{5}{|c|}{ Length of GGW (cm) } \\
\cline { 2 - 6 } & $\mathbf{4 0}$ & $\mathbf{6 0}$ & $\mathbf{8 0}$ & $\mathbf{1 0 0}$ & $\mathbf{1 2 0}$ \\
\hline $\mathbf{K}_{\mathbf{1}}$ & $\mathbf{6 . 4 7 7 9}$ & 1.9656 & 0.8936 & 6.3706 & 464.80 \\
\hline $\mathbf{K}_{\mathbf{2}}$ & 0.7358 & 0.9343 & 1.3548 & 0.6780 & -1.8231 \\
\hline $\mathbf{K}_{\mathbf{3}}$ & 0.2515 & -0.1052 & -0.5151 & 0.0689 & 3.8037 \\
\hline $\mathbf{K}_{\mathbf{4}}$ & 0.2515 & -0.1052 & -0.5151 & 0.0689 & 3.8037 \\
\hline $\mathbf{K}_{\mathbf{5}}$ & 0.0127 & 0.1709 & 0.1603 & 0.2531 & -0.9806 \\
\hline $\mathbf{K}_{\mathbf{6}}$ & 0.0264 & 1.0197 & 0.5734 & -2.0914 & -2.0914 \\
\hline $\mathbf{R}^{\mathbf{2}}$ & 0.905 & 0.853 & 0.803 & 0.812 & 0.950 \\
\hline
\end{tabular}




\section{Conclusion:-}

The study of relationships that combine parameters of flow through and over weir with porous body are generally complicated and choice of parameters that represent any phenomena like energy dissipation is important to get an acceptable numerical expression. The goals of the study may be conclude as:-

1. The difference in energy, $\Delta \mathrm{E}$, between upstream and downstream sides of gravel gabion weir, GGW, is directly proportional with increasing of distance of formation of hydraulic jump, DHJ, away from the weir toe in general.

2. The oscillatory effect of diameter of gravel sample used, d, appears clearly with increasing of length of the gabion weir, LT, where it is directly proportional with increasing of values of both of $\Delta \mathrm{E}$ and $\mathrm{DHJ}$ for small length, $(\mathrm{LT}=40 \mathrm{~cm})$, while fluctuates with transition from small to medium lengths, $(\mathrm{LT}=60 \mathrm{~cm})$, and returns to proportion inversely with increasing of values of both criterions for medium and large lengths.

3. At the minimum value of applied discharge, $\mathrm{q}_{\text {min. }}$, the effect of gradation of $\mathbf{d}$ appears clearly on the value of DHJ for the small length, where DHJ approaches to the weir toe as the value of $d$ increases, after that the gradation has a light effect for the medium length, $(\mathrm{LT}=60 \mathrm{~cm})$, where the values of DHJ approaches from each other within a short range, and then the gradation effect is gradually decreasing noticeably at the forth length, $(\mathrm{LT}=100 \mathrm{~cm})$, till it disappears at the large length, $(\mathrm{LT}=120 \mathrm{~cm})$. Therefore, there is no formation to the hydraulic jump for all gravel samples.

4. At the comparison value of applied discharge, $\mathrm{q}_{\mathrm{cmpr}}$, the effect of gradation of $\mathbf{d}$ is clear on DHJ values for all lengths, where DHJ approaches from the toe of GGW as the value of $\mathbf{d}$ increases, but this effect starts to be less for the last three samples of gravel used and for the last three lengths DHJ values approaches from each other within a short range.

5. At the maximum value of discharge applied within every series of test runs, $\mathrm{q}_{\max }$, the gradation of $\mathbf{d}$ on DHJ appears clearly for small lengths where the hydraulic jump approaches to the toe of GGW with increasing of value of $\mathbf{d}$, while the gradation oscillates for the medium lengths, and then its effect returns to appear for large length as in small length.

6. At $\mathrm{q}_{\min }$, the value of $\Delta \mathrm{E}$ decreases by increasing of $\mathbf{d}$ value for both small and medium lengths, and fluctuates for large length.

7. At $\mathrm{q}_{\mathrm{cmpr}}$, the value of $\Delta \mathrm{E}$ decreases by increasing of $\mathbf{d}$ value for all lengths.

8. At $\mathrm{q}_{\max }$, the value of $\Delta \mathrm{E}$ decreases by increasing of both of $\mathbf{d}$ and $\mathrm{q}_{\max }$ values for small length and fluctuates for both parameters and medium lengths, and then returns to take the same behavior of small length for large length.

\section{References:-}

1. A. E. Larson, "Energy Dissipation in Culverts by Forcing a Hydraulic Jump at The Outlet," M. Sc., in Civil Engineering, Thesis, Washington State University, Washington, USA, 2004.

2. A. F. Eloubaidy, J. H. Al-Baidhani, and A. H. Ghazali, "Dissipation of Hydraulic Energy by Curved Baffle Blocks," Pertanika Journal of Science and Technology, Vol. 7, No. 1, 1999, pp. 69-77.

3. B. Li, V. K. Garga, and M. H. Davies, "Relationships for Non-Darcy Flow in Rockfill," Journal of Hydraulic Engineering, Vol. 124, No. 2, 1998, pp. 206-212.

4. F. Salmasi, "Energy Loss at Drops Using Neurosolutions," International Journal of Environmental, Chemical, Ecological, Geological, and geophysical Engineering, Vol. 5, No. 8, 2011, pp. 458-466.

5. H. Chanson, "Broad-Crested Weir - Discussion," Journal of Irrigation and Drainage Engineering, ASCE, Vol. 121, No. 2, 1995, pp. 222-224.

6. I. A. Juma, "Determination of Coefficient of Transmissibility of Graded River Gravel," M. Sc., in Irrigation and Drainage Engineering, Thesis, University of Mosul, Mosul, 1987.

7. I. H. Mohamed, "Flow over Gabion Weirs," Journal of Irrigation and Drainage, ASCE, Vol. 136, No. 8, 2010, pp. 573-577.

8. J. A. Kells, "Spatially Varied Flow Over Rockfill Embankments," Canadian Journal of Civil Engineering, The $10^{\text {th }}$ Canadian Hydro technical Conference, Vancouver, British Colombia, Vol. 20, 1993, pp. 820-827.

9. J. C. Robarge, D. C. Gramer, and W. E. Lee, "Hydraulic of Flow through Porous Gravel Structures," Alden Research Laboratories, Holden, Massachusetts, 1976, pp. 1-18.

10. J. Kang, S. Kim, H. Yeo, and N. Lee, "Experimental Study on Flow Characteristic in Sloping Weir," Engineering 6, 2014, pp. 329-337.

11. J. R. Wyrick, and G. B. Pasternack, "Modeling Energy Dissipation and Hydraulic Jump Regime Responses to Channel Nonuniformity at River Steps," Journal of Geophysical Research, Vol. 113, F 03303, 2008, pp. 1-25. 
12. K. Michioku, S. Maeno, T. Furusawa, and M. Haneda, "Discharge Through a Permeable Rubble Mound Weir," Journal of Hydraulic Engineering, ASCE, Vol. 131, No. 1, 2005, pp. 1-10.

13. M. A. Ashour, T. Sayed, and S. El-Attar, "A New Water Energy Dissipater for Efficient Energy Dissipation and enriching the flow with Dissolved Oxygen Content," Limnol, Rev., Vol. 14, No. 1, 2014, pp. 3-11.

14. M. A. Ashour, T. Sayed, and S. El-Attar, "Impact of Curved Shaped Energy Dissipaters Downstream Diversion Head Structures to The Dissolved Oxygen Content in Irrigation Canals and Enhancement of Irrigation Water Quality," International Journal of Scientific Research and Innovative Technology, Vol. 2, No. 2, 2015, pp. 14-26.

15. R. P. Curtis, and J. D. Lawson, "Flow Over and Through Rockfill Banks," Journal of Hydraulic Division, ASCE, Vol. 93, No. HY5, Proc., Paper 5412, 1967, pp. 1-21.

16. S. H. Ali, "Hydraulic Characteristics of flow through Rockfill Dykes," M. Sc. in Irrigation and Drainage Engineering, Thesis, University of Mosul, Mosul, 1985.

17. S. K. Gupta, R. C. Mehta, and V. K. Dwivedi, "Modeling of Relative Length and Relative Energy Loss of Free Hydraulic Jump in Horizontal Prismatic Channel," Chemical, Civil, and Mechanical Engineering Traks. of the $3^{\text {rd }}$ Nirma University International Conference on Engineering, Vol. 1, No. 75, 2013, pp. 529-537.

18. S. M. Hosseini, "On The Performance of Different Empirical Loss Equations for Flow Through Coarse Porous Media," IJE Transetions B: Applications, Vol. 15, No. 3, 2002, pp. 249-254.

19. S. Pagliara, R. Das, and M. Palermo, "Energy Dissipation on Submerged Block Ramps," Journal of Irrigation and Drainage Engineering, Vol. 134, No. 4, 2008, pp. 527-532.

20. T. Morii, "Head Loss Equation to Predict Flow Transmissibility of Rockfill structures, "Proc. of the $5^{\text {th }}{ }^{\mathrm{I}} \mathrm{CHE}$, 2002, pp. 208.

21. Y. A. Moussa, A. M. Ali, and Y. K. Saleh, "Performance of Sills Over Aprons under The Effect of Submerged Hydraulic Jump, (Case Study: Naga Hammadi Barrage)," Ain Shams Engineering Journal, Production and Hosting by Elsevier, 2016.

22. Y. Kim, G. Choi, H. Park, and S. Byeon, "Hydraulic Jump and Energy Dissipation with Sluice Gate, mdpi/Journal/wate, 7, 2015, pp. 5115-5133.

23. Z. Ahmed, N. M. Petappa, and B. Westrich, "Energy Dissipation on Block Ramps with Staggered Boulders," Journal of Hydraulic Engineering, Vol. 135, No. 6, 2009, pp. 522-526. 